\title{
Nur auf Stundenbasis machen Einkommensvergleiche Sinn
}

\section{Christoph Bosshard}

Dr. med., Vizepräsident der FMH, Departementsverantwortlicher Daten, Demographie und Qualität

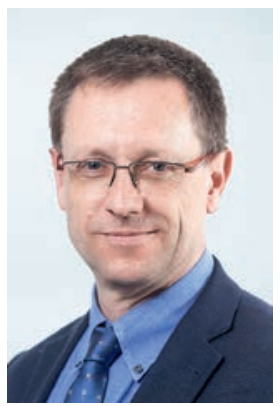

Transparenz über die Ärzte-Einkommen hat bei der FMH Tradition: Während Jahrzehnten publizierte die FMH in der Schweizerischen Ärztezeitung jedes Jahr die Einkommensstudie der Ärzteschaft, bis das Bundesamt für Sozialversicherungen im Jahr 2013 aufgrund veränderter rechtlicher Rahmenbedingungen den für die Studie notwendigen Bezug der AHV-Einkommensdaten von der Ausgleichskasse untersagte. Gleichzeitig monierte das Bundesamt, die Verwendung der AHV-Einkommen lasse zu wenig validierbare Rückschlüsse auf die reale Einkommenssituation zu, was die FMH auch stets als Schwäche der Studie ausgewiesen hatte.

\section{Um eine differenzierte Vergleichsgrösse zu} haben, hat die FMH eine Studie in Auftrag gegeben, um die Stundenlohnfrage zu klären.

Nun gut, so überlegten wir uns, ob und wie in dieser Sache weiter zu verfahren sei. Der Ansatz der Datenerhebung MAS eines anderen Bundesamtes, nämlich desjenigen für Statistik, zeigte schliesslich besseres Potential. Mit der erwartet grossen Professionalität dieses Partners in der Erhebung und im Umgang mit Daten war für die FMH die Grundlage für eine konstruktive Vorgehensweise gegeben. Die erste Erhebung, welche die Daten von 2015 betraf, konnte Mitte 2017 abgeschlossen werden und darf in allen Belangen als Erfolg gewertet werden. Die hohe Teilnahmequote ermöglichte die gewünschten statistischen Analysen und Aussagen.

Ein weiterer Nachteil der AHV-Einkommen ist, dass aus der Zahl nicht ersichtlich wird, welches Einkommen aus medizinischer Tätigkeit erwirtschaftet wird. Im Gegensatz dazu geben die Zahlen der MAS-Erhebung differenziert Auskunft über den Ertrag einer Praxis, den Aufwand (Kostensatz) und somit über das Einkommen einer selbständigen Ärztin oder eines Arztes. Für die FMH ist das überwiegende öffentliche Interesse im Bereich der sozialversicherungsbasierten Einkommen unbestritten. Die MAS-Zahlen zeigen nun, welche Lohnteile aus den Sozialversicherungen generiert werden, wie viel aus Zusatzversicherungen stammt und welche Einkommen mit weiteren medizi- nischen Tätigkeiten generiert werden, zum Beispiel mit Gutachten. Denn Ärztinnen und Ärzten steht es weiter frei, auch ausserhalb des Gesundheitswesens Verdienstmöglichkeiten wahrzunehmen. Die Zahlen der MAS-Erhebung wurden am 10. April 2018 publiziert und zeigen, dass das durchschnittliche Betriebsergebnis einer Einzelpraxis im Jahr 2015 CHF 155000 betrug, wobei der sozialversicherungsbasierte Anteil CHF 138000 umfasste. Dies sind im gesamtschweizerischen Durchschnitt ansprechende Einkommen. Nur drängen sich hier einige Fragen auf, welche zu klären sind, bevor man Vergleiche anstellt: Mit welchem Arbeitsstundeneinsatz werden diese Einkommen generiert? In welcher Lebensphase und wie lange können solche Pensen geleistet werden? Die FMH wollte hier Klarheit schaffen und hat sich der Stundenlohnfrage angenommen. Mein Dank geht an dieser Stelle an die Autorenschaft der in dieser Ausgabe vorgestellten Studie, welche einen grossen Schritt in Richtung besserer Transparenz ermöglicht. Als Grundlage dient wiederum eine Erhebung des BFS. Das Resultat ist deutlich: Die Ärzteschaft verpasst die Podestplätze und landet mit einem Stundenlohn von CHF 67 auf dem 4. Rang hinter den Zahnärzten, Anwälten und Volkswirten. Das Fazit ist klar: Das relativ hohe Jahreseinkommen von Ärztinnen und Ärzten im Vergleich zu anderen Berufsgruppen entsteht durch überdurchschnittlich lange Arbeitszeiten. Bisweilen ist es eben notwendig, etwas genauer hinzuschauen, wenn differenzierte Vergleiche gefragt sind. Die FMH wird sich auch weiterhin für Transparenz und

\section{Das vergleichsweise hohe Jahreseinkommen} von Ärztinnen und Ärzten entsteht durch überdurchschnittlich lange Arbeitszeiten.

faire Vergleiche einsetzen! Damit die Ärzteschaft, aber auch die Politik und Verwaltung über eine verlässliche und fundierte Datengrundlage verfügt, empfiehlt der Zentralvorstand der FMH den Mitgliedern, Arztpraxen und ambulanten Zentren an der aktuell laufenden MAS-Erhebung 2017 des Bundesamtes für Statistik teilzunehmen. 